\title{
FRICTIONAL HEATING DURING SLIDING OF TWO SEMI-SPACES WITH ARBITRARY THERMAL NONLINEARITY
}

\author{
Ewa OCH \\ Faculty of Mechanical Engineering, Bialystok University of Technology, 45C Wiejska Street, Bialystok, 15-351, Poland \\ e.och@doktoranci.pb.edu.pl

\begin{abstract}
Analytical and numerical solution for transient thermal problems of friction were presented for semi limited bodies made from thermosensitive materials in which coefficient of thermal conductivity and specific heat arbitrarily depend on the temperature (materials with arbitrary non-linearity). With the constant power of friction assumption and imperfect thermal contact linearization of nonlinear problems formulated initial-boundary thermal conductivity, using Kirchhoff transformation is partial. In order to complete linearization, method of successive approximations was used. On the basis of obtained solutions a numerical analysis of two friction systems in which one element is constant (cermet FMC-845) and another is variable (grey iron ChNMKh or aluminum-based composite alloy AL MMC) was conducted.
\end{abstract}

Key words: Frictional Heating, Arbitrary Thermal Non-Linearity, Thermosensitive Materials

\section{INTRODUCTION}

Developing analytical or analytical - numerical solutions of heat conduction problems with regard to the frictional heating is necessary in designing new types of friction nodes (eg. the choice of friction material, estimation of the temperature level, selection of the process operational parameters, etc.). In such modeling method the real friction pairs are replaced by systems such as: strip - halfspace, strip - strip or semi-space - semi-space.

Friction heat problem solution for a homogeneous semi-space sliding at constant speed on the surface of the second semi-space, with constant thermophysical materials properties and perfect thermal contact condition fulfillment are presented in Grylitsky (1996) monograph, and solution taking into account imperfect thermal contact - in work: Sazonov (2008).

Frictional heating during two semi-space, strip and semi-space, slipping with a constant delay and the two strips system with regard to various kinds of conditions of thermal contact was examined in works: Nosko et al. (2009), Yevtushenko et al. (2013, 2014), Barber (1976). Details on methods for solving one-dimensional thermal problems of friction with constant thermophysical materials properties are given in monograph Kuciej (2012).

The analysis of literature sources concerning analytical or analytical-numerical modeling of heat generation processes due to frictional forces, shows that models considering constant thermophysical properties of friction materials are very well developed. Whereas, solutions involving a non-linearity of frictional materials pairs have been so far developed in an insufficient number of cases.

Homogeneous semi-space surface frictional heating with linear dependence of thermal conductivity coefficients and specific heat of the temperature at a constant ratio of the thermal diffusivity (material with a simple non-linearity) was examined in the work: AbdelAal, (1997), Abdel-Aal et al. (1997), Abdel-Aal and Smith (1998, 1998b). Methods of solving one-dimensional initial-boundary problems of thermal conductivity for the two semi-spaces sliding against one another at a constant linear velocity or semi-space made of materials with a simple non-linearity, are proposed in the work: Och (2013), Evtushenko et al. (2014), Yevtushenko et al. (2014, 2014b), whereas solutions involving arbitrary non-linearity (thermophysical properties of materials change under the influence of temperature in any way) in the work: Yevtushenko et al. (2014c, d).

Surveys of analytical and numerical methods for solving initialboundary problems of heat conduction for materials with temperature-dependent thermal properties are presented in the work: Kushnir and Popovych (2011), Yevtushenko and Kuciej (2012).

The present work is a continuation of studies presented in article Och (2013), where case of simple thermal nonlinearity of materials was considered. Whereas in this work friction elements materials are characterized by arbitrary non-linearity.

\section{STATEMENT OF THE PROBLEM}

Let the two semi-limited thermally sensitive bodies with the same initial temperature $T_{0}$ be compressed at infinite and constant pressure $p_{0}$ in the parallel direction to the $z$ axis of the Cartesian coordinate system $O x y z$ (Fig. 1). At initial point of time $t=0$ bodies start to slip with a constant speed $V_{0}$ in the positive direction of $y$-axis. Due to the friction forces, on the contact surface $z=0$ heat is generated, which penetrates into the contacting bodies - heating them.

It was assumed, that the sum of heat fluxes intensities directed perpendicularly from the contact surfaces to the inside of each contacting body, is equal to the specific power of friction forces $q_{0}=$ $f V_{0} p_{0}$ (Yevtushenko and Kuciej, 2012). Whereas the heat transfer through the surface of the friction takes place with constant coefficient of contact thermal conductivity $h$ (Podstrigach, 1963; Barber, 1970).

Further in these article, all values referring to the upper and lower semi-spaces will respectively have subscripts 1 and 2 . 


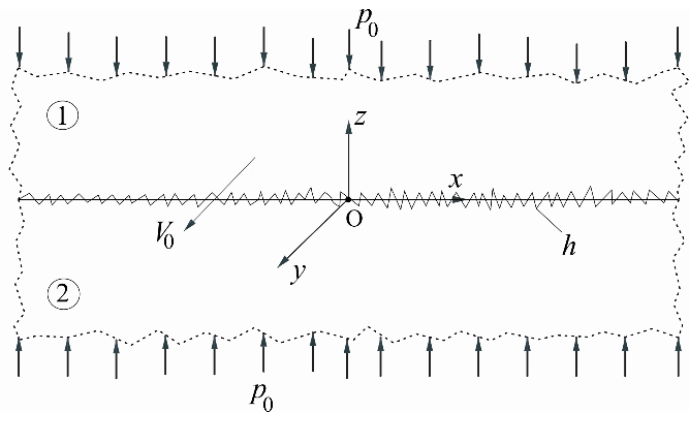

Fig. 1. The scheme of frictional heating

Both, coefficient of thermal conductivity $K_{l}$ and specific heat $c_{l}$, $l=1,2$ of the two semi-spaces materials depend on temperature $T:$

$K_{l}(T)=K_{l, 0} K_{l}^{*}(T), K_{l, 0} \equiv K_{l}\left(T_{0}\right)$

$c_{l}(T)=c_{l, 0} c_{l}^{*}(T), c_{l, 0} \equiv c_{l}\left(T_{0}\right)$

$K_{l}^{*}(T), c_{l}^{*}(T)$ - dimensionless functions. Densities $\rho_{l}, l=1,2$ of considered bodies materials are constant.

Taking into account mentioned above assumptions, the distribution of transient temperature field $T(z, t)$ in semi-spaces is found from the following heat conduction problem boundaryvalue solution:

$\frac{\partial^{2} T_{1}^{*}}{\partial \zeta^{2}}=\frac{1}{k_{0}^{*} k_{1}^{*}\left(T_{1}^{*}\right)} \frac{\partial T_{1}^{*}}{\partial \tau}, \quad \zeta>0, \tau>0$

$\frac{\partial^{2} T_{2}^{*}}{\partial \zeta^{2}}=\frac{1}{k_{2}^{*}\left(T_{2}^{*}\right)} \frac{\partial T_{2}^{*}}{\partial \tau}, \quad \zeta<0, \tau>0$

$\left.K_{2}^{*}\left(T_{2}^{*}\right) \frac{\partial T_{2}^{*}}{\partial \zeta}\right|_{\zeta=0^{-}}-\left.K_{0}^{*} K_{1}^{*}\left(T_{1}^{*}\right) \frac{\partial T_{1}^{*}}{\partial \zeta}\right|_{\zeta=0^{+}}=1, \tau>0$

$\left.K_{2}^{*}\left(T_{2}^{*}\right) \frac{\partial T_{2}^{*}}{\partial \zeta}\right|_{\zeta=0^{-}}+\left.K_{0}^{*} K_{1}^{*}\left(T_{1}^{*}\right) \frac{\partial T_{1}^{*}}{\partial \zeta}\right|_{\zeta=0^{+}}=$

$B i\left[T_{1}^{*}\left(0^{+}, \tau\right)-T_{2}^{*}\left(0^{-}, \tau\right)\right], \tau>0$$$
T_{l}(\zeta, \tau) \rightarrow T_{0}^{*},|\zeta| \rightarrow \infty, \quad l=1,2
$$$$
T_{l}(\zeta, 0)=T_{0}^{*},|\zeta|<\infty, l=1,2
$$

where:

$\zeta=\frac{z}{a}, \quad \tau=\frac{k_{2} t}{a^{2}}, \quad K_{0}^{*}=\frac{K_{1,0}}{K_{2,0}}, \quad k_{0}^{*}=K_{0}^{*} \frac{\rho_{2} c_{2,0}}{\rho_{1} c_{1,0}}, B i=\frac{h a}{K_{2,0}}$

$T_{a}=\frac{q_{0} a}{K_{2,0}}, \quad T_{l}^{*}=\frac{T_{l}}{T_{a}}, \quad T_{0}^{*}=\frac{T_{0}}{T_{a}}, l=1,2$

$k_{l}^{*}\left(T_{l}^{*}\right)=\frac{K_{l}^{*}\left(T_{l}^{*}\right)}{c_{l}^{*}\left(T_{l}^{*}\right)}, l=1,2$

$a-$ is a characteristic linear dimension. Further, as the value of this parameter, we take the effective depth of heat penetration, i.e. the distance from the contact surface, on which the temperature is equal to $5 \%$ of the maximal temperature on the surface of friction (Chichinadze et al., 1979).

\section{LINEARIZATION OF THE PROBLEM}

In order to linearize the non-linear boundary-value heat conduction problem of friction (2) - (7) we introduce the Kirchhoff's function (Kirchhoff, 1894):

$\Theta_{l}(\zeta, \tau)=\int_{T_{0}^{*}}^{T^{*}} K_{l}^{*}\left(T_{l}^{*}\right) d T_{l}^{*}, l=1,2$
As a result, we obtained a sequence of linear boundary-value problems in relation to the functions $\Theta_{l}^{(i)}(\zeta, \tau), i=0,1, \ldots$, that are successive approximations of the sought Kirchhoff's function (Yevtushenko et al., 2014c, d):

$\frac{\partial^{2} \Theta_{1}^{(i)}}{\partial \zeta^{2}}=\frac{1}{k_{0}^{*} k_{1}^{*(i)}} \frac{\partial \Theta_{1}^{(i)}}{\partial \tau}, \zeta>0, \tau>0$

$\frac{\partial^{2} \Theta_{2}^{(i)}}{\partial \zeta^{2}}=\frac{1}{k_{2}^{*(i)}} \frac{\partial \Theta_{2}^{(i)}}{\partial \tau}, \zeta<0, \tau>0$

$\left.\frac{\partial \Theta_{2}^{(i)}}{\partial \zeta}\right|_{\zeta=0}-\left.K_{0}^{*} \frac{\partial \Theta_{1}^{(i)}}{\partial \zeta}\right|_{\zeta=0}=1, \tau>0$

$\left.\frac{\partial \Theta_{2}^{(i)}}{\partial \zeta}\right|_{\zeta=0}+\left.K_{0}^{*} \frac{\partial \Theta_{1}^{(i)}}{\partial \zeta}\right|_{\zeta=0}=$

$B i^{(i)}\left[\Theta_{1}^{(i)}(0, \tau)-\Theta_{2}^{(i)}(0, \tau)\right], \tau>0$

$\Theta_{l}^{(i)}(\zeta, \tau) \rightarrow 0, \quad|\zeta| \rightarrow \infty, \quad \tau \geq 0, \quad l=1,2$

$\Theta_{l}^{(i)}(\zeta, 0)=0, \quad|\zeta|<\infty, \quad l=1,2$

gdzie:

$k_{l}^{*(0)}=1, k_{l}^{*(i)}=k_{l}^{*}\left\{T^{*}\left[\Theta_{l}^{(i-1)}\left(\zeta^{*}, \tau^{*}\right)\right]\right\}$
$i=1,2, \ldots ; l=1,2$

$B i^{(0)}=B i$,

$B i^{(i)}=B i \frac{\left\{T^{*}\left[\Theta_{1}^{(i-1)}\left(0, \tau^{*}\right)\right]-T^{*}\left[\Theta_{2}^{(i-1)}\left(0, \tau^{*}\right)\right]\right\}}{\left[\Theta_{1}^{(i-1)}\left(0, \tau^{*}\right)-\Theta_{2}^{(i-1)}\left(0, \tau^{*}\right)\right]}, i=1,2, \ldots$,

$\left(\zeta^{*}, \tau^{*}\right)$ - these are established values of dimensionless coordinate and time (8), for which we perform iteration.

\section{KIRCHHOFF FUNCTION}

By applying the Laplace integral transform (Sneddon, 1972):

$\bar{\Theta}_{l}^{(i)}(\zeta, p) \equiv L\left[\Theta_{l}^{(i)}(\zeta, \tau) ; p\right]=\int_{0}^{\infty} \Theta_{l}^{(i)}(\zeta, \tau) e^{-p \tau} d \tau$

to the linear boundary-value problem (11)-(19), we obtain the following boundary problem for two ordinary differential equations of the second order:

$\frac{d^{2} \bar{\Theta}_{1}^{(i)}(\zeta, p)}{d \zeta^{2}}-\frac{p}{k_{0}^{*} k_{1}^{*(i)}} \bar{\Theta}_{1}^{(i)}(\zeta, p)=0, \zeta>0$

$\frac{d^{2} \bar{\Theta}_{2}^{(i)}(\zeta, p)}{d \zeta^{2}}-\frac{p}{k_{2}^{*(i)}} \bar{\Theta}_{2}^{(i)}(\zeta, p)=0, \quad \zeta<0$

$\left.\frac{d \bar{\Theta}_{2}^{(i)}}{d \zeta}\right|_{\zeta=0}-\left.K_{0}^{*} \frac{d \bar{\Theta}_{1}^{(i)}}{d \zeta}\right|_{\zeta=0}=\frac{1}{p}$

$\left.\frac{d \bar{\Theta}_{2}^{(i)}}{d \zeta}\right|_{\zeta=0}+\left.K_{0}^{*} \frac{d \bar{\Theta}_{1}^{(i)}}{d \zeta}\right|_{\zeta=0}=B i^{(i)}\left[\bar{\Theta}_{1}^{(i)}(0, p)-\bar{\Theta}_{2}^{(i)}(0, p)\right]$

$\bar{\Theta}_{l}^{(i)}(\zeta, p) \rightarrow 0, \quad|\zeta| \rightarrow \infty, \quad l=1,2$

Solution to the problem (21)-(25) takes form:

$\bar{\Theta}_{1}^{(i)}(\zeta, p)=\frac{\sqrt{k^{*(i)}} e^{-\zeta_{1}^{(i)} \sqrt{p}}}{2 \varepsilon p\left(\sqrt{p}+\beta^{(i)}\right)}\left(1+B i^{(i)} \sqrt{\frac{k_{2}^{*(i)}}{p}}\right), \zeta \geq 0$,

$\bar{\Theta}_{2}^{(i)}(\zeta, p)=\frac{e^{-\zeta_{2}^{(i)} \sqrt{p}}}{2 \varepsilon p\left(\sqrt{p}+\beta^{(i)}\right)}\left(\varepsilon+B i^{(i)} \sqrt{\frac{k_{1}^{*(i)}}{p}}\right), \zeta \leq 0$, 
$\zeta_{1}^{(i)}=\frac{\zeta}{\sqrt{k_{0}^{*} k_{1}^{*(i)}}}, \zeta_{2}^{(i)}=\frac{|\zeta|}{\sqrt{k_{2}^{*(i)}}}$,

$\beta^{(i)}=0.5 \varepsilon^{-1} B i^{(i)} m^{(i)}, \quad m^{(i)}=\sqrt{k^{*(i)}}+\varepsilon$,

$k^{*(i)}=\frac{k_{1}^{*(i)}}{k_{2}^{*(i)}}, \varepsilon=\frac{K_{0}^{*}}{\sqrt{k_{0}^{*}}}$.

Applying the inversion formulae (Bateman and Erdelyi, 1954):

$L^{-1}\left[\frac{\beta e^{-\alpha \sqrt{p}}}{p \sqrt{p}(\sqrt{p}+\beta)} ; \tau\right]=\Phi(\alpha, \tau)-\beta^{-1} \Psi(\alpha, \beta, \tau)$

$L^{-1}\left[\frac{\beta e^{-\alpha \sqrt{p}}}{p(\sqrt{p}+\beta)} ; \tau\right]=\Psi(\alpha, \beta, \tau), \alpha, \beta \geq 0$

$\Phi(\alpha, \tau)=2 \sqrt{\tau} \operatorname{ierfc}\left(\frac{\alpha}{2 \sqrt{\tau}}\right), \tau \geq 0$

$\Psi(\alpha, \beta, \tau)=\operatorname{erfc}\left(\frac{\alpha}{2 \sqrt{\tau}}\right)-e^{\alpha \beta+\beta^{2} \tau} \operatorname{erfc}\left(\frac{\alpha}{2 \sqrt{\tau}}+\beta \sqrt{\tau}\right)$

to the solutions (26)-(29), we obtain:

$\Theta_{1}^{(i)}(\zeta, \tau)=\frac{1}{m^{(i)}}\left[\Phi\left(\zeta_{1}^{(i)}, \tau\right)+\gamma_{1}^{(i)} \Psi\left(\zeta_{1}^{(i)}, \beta^{(i)}, \tau\right)\right]$,

$\zeta \geq 0, \tau \geq 0$

$\Theta_{2}^{(i)}(\zeta, \tau)=\frac{\sqrt{k^{*(i)}}}{m^{(i)}}\left[\Phi\left(\zeta_{2}^{(i)}, \tau\right)+\gamma_{2}^{(i)} \Psi\left(\zeta_{2}^{(i)}, \beta^{(i)}, \tau\right)\right]$

$\zeta \leq 0, \tau \geq 0$

$\gamma_{1}^{(i)}=\frac{\left(\sqrt{k^{*(i)}}-\varepsilon\right)}{\sqrt{k_{2}^{*(i)}}\left(\sqrt{k^{*(i)}}+\varepsilon\right) B i(i)}, \gamma_{2}^{(i)}=\frac{\varepsilon\left(\varepsilon-\sqrt{k^{*(i)}}\right)}{\sqrt{k_{1}^{*(i)}}\left(\sqrt{k^{*(i)}}+\varepsilon\right) B i(i)}$

In particular case $\zeta=0$ from solutions (34)-(36) we obtain:

$\Theta_{1}^{(i)}(0, \tau)=\frac{1}{m^{(i)}}\left[\varphi(\tau)+\gamma_{1}^{(i)} \psi\left(\beta^{(i)}, \tau\right)\right], \tau \geq 0$,

$\Theta_{2}^{(i)}(0, \tau)=\frac{\sqrt{k^{*(i)}}}{m^{(i)}}\left[\varphi(\tau)+\gamma_{1}^{(i)} \psi\left(\beta^{(i)}, \tau\right)\right]$,

$\varphi(\tau)=2 \sqrt{\frac{\tau}{\pi}}$

$\psi\left(\beta^{(i)}, \tau\right)=1-e^{\beta^{(i)^{2}} \tau} \operatorname{erfc}\left(\beta^{(i)} \sqrt{\tau}\right)$.

\section{ITERATIVE SCHEME}

To find the relation between temperature and Kirchhoff's function the form of functions $K_{l}^{*}\left(T_{l}^{*}\right)$ and $c_{l}^{*}\left(T_{l}^{*}\right)$ should be specified (1). We assume that these are polynomials:

$K_{l}^{*}\left(T_{l}^{*}\right)=\sum_{n=0}^{N_{l}} a_{l, n}\left(T_{l}^{*}\right)^{n}, c_{l}^{*}\left(T^{*}\right)=\sum_{n=0}^{M_{l}} b_{l, n}\left(T_{l}^{*}\right)^{n}$

where $a_{l, n}$ and $b_{l, n}$ - are known coefficients for materials of each considered friction pairs. We use both equations (41) to find the function $k_{l}^{*}\left(T_{l}^{*}\right)(10)$. In addition, substituting the function $K_{l}^{*}\left(T_{l}^{*}\right)$ (41) into equation (10), after integration we obtain the relation between temperature and Kirchhoff's function:

$\Theta_{l}\left(\zeta^{*}, \tau^{*}\right)=\sum_{n=0}^{n} a_{l, n}^{\prime}\left\{\left[T^{*}\left(\zeta^{*}, \tau^{*}\right)\right]^{n+1}-\left(T_{0}^{*}\right)^{n+1}\right\}$

where $a_{l, n}^{\prime}=a_{l, n} /(1+n), l=1,2$. Using the method of least squares we located dependency inverse to (42):

$T_{l}^{*}\left(\zeta^{*}, \tau^{*}\right)=\sum_{n=0}^{N_{l}} c_{l, n}\left[\Theta_{l}\left(\zeta^{*}, \tau^{*}\right)\right]^{n}, l=1,2$

where $c_{l, n}$ - are known coefficients.
For the zero approximation $\left(i=0, k_{l}^{(0)}=1, B i^{(0)}=B i\right)$ solutions (34) - (40) are coincident with boundary-value problems solutions with constant thermo-physical properties of materials (Sazonov, 2008). The relation between temperature and Kirchhoff's function at this iterative step is linear:

$T_{l}^{*(0)}\left(\zeta^{*}, \tau^{*}\right)=\Theta_{l}^{(0)}\left(\zeta^{*}, \tau^{*}\right)+T_{0}^{*}, l=1,2$.

For successive approximations $(i \geq 1)$ we begin with calculating the values of $k_{l}^{*(i)}, l=1,2,(17)$ and $B i^{(i)}(18)$ and finding the function $\Theta_{l}^{(i)}\left(\zeta^{*}, \tau^{*}\right)(34)-(36)$ or $\Theta_{l}^{(i)}\left(0, \tau^{*}\right)(37)-(40)$, $l=1,2$. Then from the formula (43), we obtain an approximation of the dimensionless temperature $T_{l}^{*(i)}\left(\zeta^{*}, \tau^{*}\right)$ or $T_{l}^{*(i)}\left(0, \tau^{*}\right)$, $l=1,2$. The convergence of an iterative process in $\left(\zeta^{*}, \tau^{*}\right)$ is monitored by checking the inequality (Euclidean norm):

$\sqrt{\left(\frac{T_{1}^{*(i)}-T_{2}^{*(i-1)}}{T_{1}^{*(i)}}\right)^{2}+\left(\frac{T_{2}^{*(i)}-T_{2}^{*(i-1)}}{T_{2}^{*(i)}}\right)^{2}} \leq 10^{-6}$.

\section{NUMERICAL ANALYSIS AND CONCLUSIONS}

The calculations have been made for the following friction pairs: gray iron ChNMKh - cermet FMC-845 and composite on aluminum alloy base AL MMC - FMC-845. These materials are used to produce friction elements of braking systems (Chichinadze et al., 1986; $\mathrm{Kim}$ el al., 2008). The values of the thermophysical properties of selected materials at temperature $T_{0}=20^{\circ} \mathrm{C}$ are given in Tab. 1 . Graphs of functions $K_{l}^{*}\left(T^{*}\right)$ and $c_{l}^{*}\left(T^{*}\right)(41)$, for the consideraded friction materials are shown in Fig. 2, and the corresponding values of the coefficients $a_{l, n}, b_{l, n}$ and $c_{l, n}, n=0,1,2,3$ are given in Tab. 2. We can notice that the coefficient of thermal conductivity of gray iron ChNMKh and cermet's FMC-845 decreases with increasing temperature, and $\mathrm{AL} M M C$ increases in the range $0 \leq T^{*} \leq 0.5$, and then for $0.5<T^{*} \leq 1.3$ decreases, and next for $T^{*}>1.3$ rise again. Specific heat of all considered materials increases with increase of temperature. Calculations were performed for the following input parameters: $q_{0}=1 \mathrm{MW} / \mathrm{m}$, $a=5 \mathrm{~mm}, T_{a}=204^{\circ} \mathrm{C}$ and $B i=5$.

Tab. 1. The thermo-physical coefficients values of the materials at the $T_{0}=20^{\circ} \mathrm{C}$

\begin{tabular}{|c|c|c|c|}
\hline Materials & $\begin{array}{c}K_{0} \\
\mathrm{~W} /(\mathrm{m} \cdot \mathrm{C})\end{array}$ & $\begin{array}{c}c_{0} \\
\mathrm{~J} /(\mathrm{kg} \cdot \mathrm{C})\end{array}$ & $\begin{array}{c}\rho \\
\mathrm{kg} / \mathrm{m}^{3}\end{array}$ \\
\hline $\begin{array}{c}\text { FMC-845 } \\
\text { (Chichinadze et al., 1986) }\end{array}$ & 24.5 & 392.2 & 6000 \\
\hline $\begin{array}{c}\text { ChNMKh } \\
\text { (Chichinadze et al., 1986) }\end{array}$ & 51 & 500.1 & 7100 \\
\hline $\begin{array}{c}\text { AL MMC } \\
\text { (Kim el al., 2008) }\end{array}$ & 155.75 & 874 & 2730 \\
\hline
\end{tabular}

Evolutions of the dimensionless temperature on the contact surface of two selected friction pairs ChNMKh-FMC-845 and AL MMC-FMC-845 are presented in Fig. 3. The calculations have been made with taking into account friction materials thermal properties changes influenced by temperature (solid lines) and at the constant thermal properties of these materials (dashed lines). 
Tab. 2. The approximation coefficients values

\begin{tabular}{|c|c|c|c|c|c|}
\hline & $n$ & 0 & 1 & 2 & 3 \\
\hline \multirow{3}{*}{ 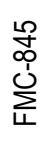 } & $a_{n}$ & 1 & 0.001799 & -0.019018 & 0.001953 \\
\hline & $b_{n}$ & 1 & 0.000547 & 0.048323 & 0.001003 \\
\hline & $c_{n}$ & 0.098041 & 0.99977 & 0.001746 & 0.005215 \\
\hline \multirow{3}{*}{ 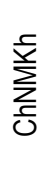 } & $a_{n}$ & 1 & -0.112 & & \\
\hline & $b_{n}$ & 1 & 0.325214 & 0.065993 & -0.027336 \\
\hline & $c_{n}$ & 0.098543 & 1.012837 & 0.05264 & 0.011315 \\
\hline \multirow{3}{*}{$\sum_{\sum}^{U}$} & $a_{n}$ & 1 & 0.356782 & -0.421682 & 0.141147 \\
\hline & $b_{n}$ & 1 & 0.209544 & -0.147707 & 0.062199 \\
\hline & $c_{n}$ & 0.097949 & 0.926329 & 0.002449 & -0.00639 \\
\hline
\end{tabular}

The temperature increases monotonically during the whole process of frictional heating, which is characteristic for the heating process at the constant power of friction forces (Grylitsky, 1996). The effect of thermal sensitivity with temperature increase is most noticeable for the cermets in the case of frictional pair ChNMKhFMC-845 (Fig. 3a). However for gray iron ChNMKh temperature difference after certain time from beginning of the frictional heating process $(\tau \approx 0.5)$, reaches predetermined value. Taking into account FMC-845 thermal sensibility results in temperature increase, and decrease in ChNMKh, compared to the temperature values found at constant thermophysical properties of materials. This is caused by cermet FMC-845 coefficient of thermal conductivity $(1 \%)$ and specific heat $(2.5 \%)$ decrease at $0 \leq \tau \leq 2$. Corresponding values for ChNMKh gray iron are $7 \%$ decrease of $K^{*}$ and $21 \%$ increase of $c^{*}$. It is a significant increase of $c^{*}$ in the gray iron semi-space (despite the $K^{*}$ decrease) which caused a slight decrease in temperature.

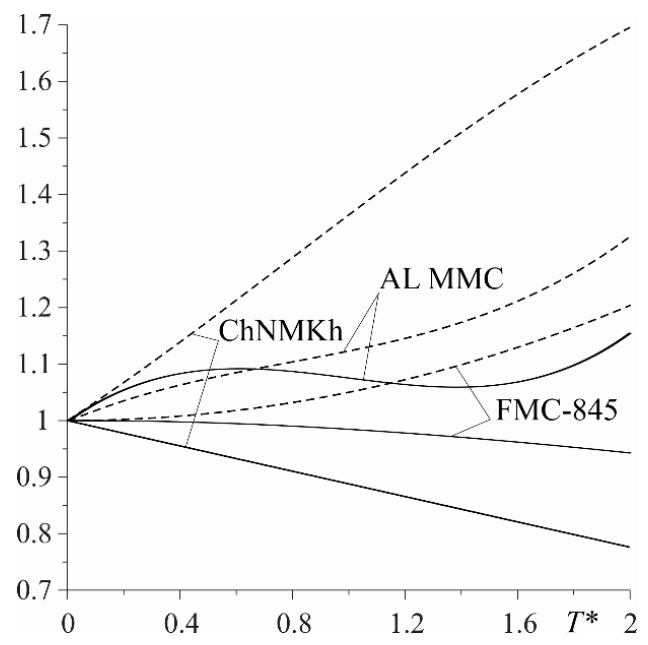

Fig. 2. Dependence of thermal conductivity $K^{*}$ (solid lines) and specific heat $c^{*}$ coefficient (dashed lines) on the dimensionless temperature $T^{*}$ for the considered materials: ChNMKh, AL MMC, FMC-845.

In the case of the second friction couple AL MMC - FMC-845 (Fig. 3b), the difference between the temperature calculated with taking into consideration nonlinearity of materials and at constant thermo-physical properties is also increasing, but much more slower than in the case of the first friction pair. Consideration of ma- terials thermal sensitivity causes a decrease in temperature on surfaces of both semi-spaces. For an AL MMC aluminum alloy $10 \%$ increase in coefficient of thermal conductivity $K^{*}$ and $6 \%$ increase in specific heat $c^{*}$ at $0 \leq \tau \leq 2$ causes, that the temperature difference calculated with and without taking into consideration the thermal sensitivity is significantly greater than cermet FMC-845.

In both considered cases, the temperature on semi-spaces surface made from cermet FMC-845 is always higher than the temperature on gray iron ChNMKh semi-spaces surface or aluminum alloy base composite AL MMC. This is due to significantly worst inferior thermal conductivity of FMC-845 when compared to ChNMKh and AL MMC (Tab. 1 and 2).

a)

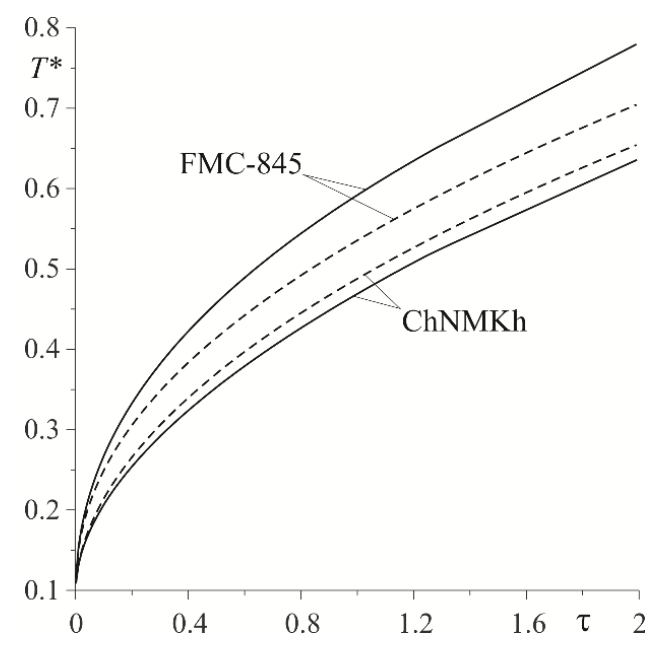

b)

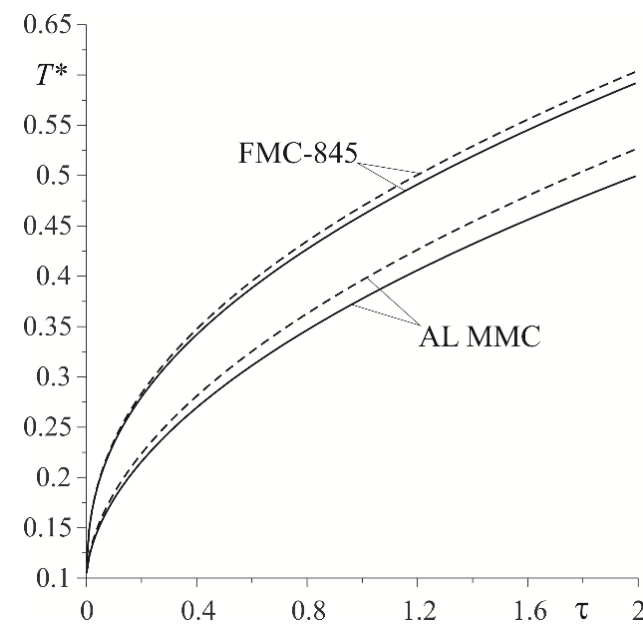

Fig. 3. Evolution of the dimensionless temperature on the contact surface for two friction pairs at $B i=5$ : a) ChNMKh - FMC-845, b) AL MMC - FMC-845 (solid lines - calculations with considered materials thermal sensitivity; dashed lines - without materials thermal sensitivity consideration

Nomenclature: $a$ - characteristic dimension; $B i$ - Biot number; $c$ - specific heat; $c_{0}-$ specific heat at an initial temperature; $\operatorname{ierfc}(x)=$ $\pi^{-1 / 2} e^{-x^{2}}-x \operatorname{erfc}(x), \operatorname{erfc}(x)=1-\operatorname{erf}(x), \operatorname{erf}(x)-$ Gauss error function; $f$ - friction coefficient; $h$ - coefficient of thermal conductivity of contact; $K$ - coefficient of thermal conductivity; $K_{0}$ - coefficient of thermal conductivity at an initial temperature; $k$ - coefficient of thermal diffusivity; $p_{0}$ - pressure; $q_{0}$ - specific power of friction; $T$ - temperature; $T_{0}$ - initial temperature; $T^{*}$-dimensionless temperature; $t$ - time; $V_{0}$ - sliding speed; $z$ - spatial coordinate; $\Theta$ - Kirchhoff's variable; $\rho$ - specific density; $\tau$ Fourier number; $\zeta$ - dimensionless spatial coordinate. 


\section{REFERENCES}

1. Abdel-Aal H. A. (1997), On the distribution of friction-induced heat in the dry sliding of metallic solid pairs, Int. Comm. Heat Mass Trans., Vol. 24, 989-998.

2. Abdel-Aal H. A., Smith S. T. (1998a), Thermal compatibility of metallic pairs in sliding contact, Int. Comm. Heat Mass Trans., Vol. 25, 599-608.

3. Abdel-Aal H. A., Smith S. T. (1998b), On friction-induced temperatures of rubbing metallic pairs with temperature-dependent thermal properties, Wear, Vol. 216, 41-59.

4. Abdel-Aal H. A., Smith S.T., Patten J. A. (1997), On the development of surface temperatures in precision single-point diamond abrasion of semiconductors, Int. Comm. Heat Mass Trans., Vol. 24, 1131-1140.

5. Barber J.R. (1970), The conduction of heat from sliding solids, Int. J. Heat. Mass Tran., Vol. 13, 857-869.

6. Barber J. R. (1976), Some thermoelastic contact problems involving frictional heating, Quarterly Journal of Mechanics and Applied Mathematics, Vol. 29, 1-13.

7. Bateman H., Erdelyi A. (1954), Tables of integral transforms, V. 1, McGraw-Hill, New York.

8. Chichinadze A. V., Matveevski R. M., Braun E. P. (1986), Materials in tribotechnics non-stationary processes, Nauka, Moscow (in Russian).

9. Chichinadze A. V., Braun E. D., Ginsburg A. G., Ignat'eva Z. V. (1979), Calculation, Test and Selection of Frictional Couples, Nauka, Moscow (in Russian).

10. Evtushenko O, Kuciej M., Och E. (2014), Influence of the thermal sensivity of materials on the temperature at friction, Mat. Sci., Vol. 50, 117-122.

11. Grylitsky D. V. (1996), Thermoelastic contact problems in tribology, A Textbook, Institute of the Contents and Methods of Education, Ministry of Education of the Ukraine, Kiev.

12. Kim S. W., Park K., Lee S. H., Kang K. H., Lim K. T. (2008), Thermophysical properties of automotive metallic brake disk materials, Int. J. Thermophys., Vol. 29, 2179-2188.

13. Kirchhoff G. R. (1894), Heat theory lectures B.G. Teubner, Leipzig (in Germany).

14. Kuciej M. (2012), Analytical models of transient frictional heating, Oficyna Wydawnicza Politechniki Białostockiej, Białystok (in Polish).

15. Kushnir R. M., Popovych V. S. (2011), Heat conduction problems of thermosensitive solids under complex heat exchange, Heat conduction - Basic Research, V. Vikhrenko Ed., In Tech, Croatia, 131-154.

16. Nosko A. L., Belyakov N. S., Nosko A. P. (2009), Application of the generalized boundary condition to solving thermal friction problems, J. Frict. Wear, Vol. 30, 615-625.
17. Och E. (2013), Frictional Heating During Sliding of Two Semi-Spaces with Simple Thermal Nonlinearities, Acta Mech. Autom., Vol. 7 , 236-240.

18. Podstrigach Ya. S. (1963), The temperature field in a system of rigid bodies coupled by thin interface, Inzh.-Fiz. Zh., Vol. 6, No 10, 129-136, (in Russian).

19. Sazonov V. S. (2008), Nonideal contact problem of nonstationary heat conduction for two half-spaces, J. Eng. Phys. Thermophys., Vol. 81, 397-408.

20. Sneddon I. N. (1972), The use of integral transforms, McGraw-Hill, New York.

21. Yevtushenko A., Kuciej M., Och E. (2014a), Effect of Thermal Sensitivity of Materials of Tribojoint on Friction Temperature, J. Frict. Wear, Vol. 35, 77-83.

22. Yevtushenko A., Kuciej M., Och E. (2014b), Influence of thermal sensitivity of the pad and disk materials on the temperature during braking, Int. Comm. Heat Mass Transf., Vol. 55, 84-92.

23. Yevtushenko A., Kuciej M., Och E. (2014c), Temperature in thermally nonlinear pad-disk brake system, Int. Comm. Heat Mass Transf., Vol. 57, 274-281.

24. Yevtushenko A., Kuciej M., Och E. (2014d), Some methods for calculating the temperature during friction of the thermosensitive materials, Num. Heat Transf., Vol. 67, 696-718.

25. Yevtushenko A. A., Kuciej M. (2012), One-dimensional thermal problem of friction during braking: The history of development and actual state, Int. J. Heat Mass Tran., Vol. 55, 4118-4153.

26. Yevtushenko A. A., Kuciej M., Yevtushenko O. (2013), The boundary conditions on the sliding surface in one-dimensional transient heat problem of friction, Int. J. Heat Mass Trans., Vol. 59, No 1, 1-8.

27. Yevtushenko A. A., Kuciej M., Yevtushenko O. (2014), The asymptotic solutions of heat problem of friction for a three-element tribosystem with generalized boundary conditions on the surface of sliding, Int. J. Heat Mass Trans., Vol. 70, 128-136.

28. Yune Y. G., Bryant M. D. (1988), Transient nonlinear thermal runaway effects in carbon graphite electrical brushers, IEEE Trans. on Components, Hybrids, and Manufacturing Technology, Vol. 11, 91-100.

29. Yune Y. G., Bryant M. D. (1989), Thermal evolution of hot spots in thermally nonlinear carbon graphite sliders, Trans. ASME. J. Tribology, Vol. 111, 591-596.

The present paper is financially supported by the National Science Centre of Poland (project No 2011/01/B/ST8/07446). 